\title{
Safety and rate of delayed adverse events with lumen-apposing metal stents (LAMS) for pancreatic fluid collections: a multicenter study
}

\section{다 $(9) \Theta$}

\section{Authors}

Dennis Yang ${ }^{1}$, Yaseen B. Perbtani ${ }^{1}$, Lazarus K Mramba², Tossapol Kerdsirichairat ${ }^{3}$, Anoop Prabhu ${ }^{3}$, Amar Manvar $^{4}$, Sammy Ho ${ }^{4}$, Davindebir Pannu ${ }^{5}$, Rajesh N. Keswani ${ }^{5}$, Daniel S. Strand ${ }^{6}$, Andrew Y. Wang ${ }^{6}$, Eduardo Quintero ${ }^{7}$, Jonathan M. Buscaglia7, Thiruvengadam Muniraj ${ }^{8}$, Harry R. Aslanian ${ }^{8}$, Peter V. Draganov ${ }^{1}$, Ali S. Siddiqui ${ }^{9}$

Institutions

1 Division of Gastroenterology and Hepatology, University of Florida, Gainesville, Florida, United States

2 Department of Biostatistics, University of Florida, Gainesville, Florida, United States

3 Division of Gastroenterology and Hepatology, University of Michigan, Ann Arbor, Michigan, United States

4 Division of Gastroenterology and Hepatology, Montefiore Medical Center, Bronx, New York, United States

5 Division of Gastroenterology and Hepatology, Northwestern University Medical Center, Chicago, Illinois, United States

6 Division of Gastroenterology and Hepatology, University of Virginia, Charlottesville, Virginia, United States

7 Division of Gastroenterology and Hepatology, Stony Brook University Medicine, Stony Brook, New York, United States

8 Section of Digestive Diseases, Yale University, New Haven, Connecticut, United States

9 Division of Gastroenterology and Hepatology, Thomas Jefferson University, Philadelphia, Pennsylvania, United States

submitted 24.4.2018

accepted after revision 24.7.2018

Bibliography

DOI https://doi.org/10.1055/a-0732-502 |

Endoscopy International Open 2018; 06: E1267-E1275

(c) Georg Thieme Verlag KG Stuttgart · New York

ISSN 2364-3722

Corresponding author

Dennis Yang, MD, Division of Gastroenterology, University of Florida, 1329 SW 16th Street, Suite 5251, Gainesville, FL 32608, USA

Fax: +1-352-627-9002

Dennis.Yang@medicine.ufl.edu

\section{ABSTRACT}

Background and study aims Endoscopic drainage with dedicated lumen-apposing metal stents (LAMS) is routinely performed for symptomatic pancreatic fluid collections (PFCs), walled-off necrosis (WON) and pseudocyst (PP). There has been increasing concern regarding delayed adverse events associated with the indwelling LAMS.

Patients and methods Multicenter retrospective analysis of consecutive patients who underwent endoscopic ultrasound (EUS)-guided LAMS placement for PFC from January 2010 to May 2017. Main outcomes included: (1) resolution of the PFC, (2) rate of delayed adverse events at follow-up, and (3) predictors of treatment failure and delayed adverse events on logistic regression.

Results A total of 122 patients (mean age 50.9 years, $68 \%$ male) underwent LAMS insertion for 64 WON (98.4\%) and 58 PP (98.3\%). PFC mean size was $10.6 \mathrm{~cm}$. PFC resolution was significantly lower for WON (62.3\%) vs. PP (96.5\%) $(P<0.001)$ on imaging at a median of 4 weeks. Stent occlusion was identified in $18(29.5 \%)$ and $10(17.5 \%)$ patients with WON and PP, respectively $(P=0.13)$. There were no cases of delayed bleeding or buried stent on follow-up endoscopy. Use of electrocautery-enhanced LAMS was the only factor associated with treatment failure of WON (OR = 13.2; $95 \%$ ci: $3.33-51.82, P=0.02$ ) on logistic regression. There were no patient, operator, or procedure-related factors predictive of stent occlusion.

Conclusions EUS-guided LAMS for PFC is associated with a low incidence of delayed adverse events. While nearly all PPs resolve at 4 weeks permitting LAMS removal shortly thereafter, many WON persist, with use of electrocauteryenhanced LAMS being the sole predictor of treatment failure. 


\section{Introduction}

Endoscopic transmural drainage is now first-line therapy for management of peripancreatic fluid collections (PFCs) given its similar efficacy, yet shorter recovery times, lower adverse event rate, and improved cost-effectiveness when compared to surgery [1,2]. Dedicated lumen-apposing metal stents (LAMS) recently have been developed to simplify endoscopic PFC drainage. The "saddle"-shaped LAMS with its wide flanges is designed to impart anchorage across the fistulous tract, thereby reducing risk of migration and peritoneal leakage; whereas the wider stent diameter is aimed at enhancing drainage, particularly of walled-off necrosis (WON) [3].

Since its introduction, numerous studies have emerged supporting the technical feasibility, efficacy and short-term safety of LAMS; with PFC resolution rates reported between $85 \%$ to $100 \%$ and early adverse events occurring in $5 \%$ to $10 \%$ of cases [4-7]. To further improve ease of use, a novel electrocauteryenhanced LAMS delivery system (Hot Axios, Boston Scientific Corporation, Marlborough, Massachusetts, United States) was recently developed. The electrocautery-enhanced tip facilitates passage of the LAMS deployment device without need for dilation of the fistulous tract. This one-step approach has streamlined the procedure and further helped disseminate the widespread practice of endoscopic PFC drainage.

Traditionally, following LAMS placement, interval imaging is obtained around 4 to 6 weeks to assess for treatment response and to guide timing of stent removal or additional interventions. However, recent data raise concern for potential delayed adverse events (AEs) associated with the indwelling LAMS.Indeed, several studies have reported on incidence of delayed bleeding and the phenomenon of "buried stent syndrome," in which extensive mucosal tissue overgrowth around the proximal flange on the luminal side compromises stent removal during follow-up [8-13]. As such, some experts have recently advocated repeating imaging at a shorter interval of 3 weeks to expedite stent removal in patients in whom the PFC has resolved [13]. Characterizing the incidence and type of delayed AEs associated with LAMS is important as it affects the followup strategy in these patients after initial PFC drainage. The objective of our study was to perform a multicenter retrospective review to assess for delayed AEs associated with LAMS. A secondary aim was to identify potential predictors of treatment failure and delayed adverse events.

\section{Patients and methods}

\section{Study population}

This was a multicenter observational, retrospective, cohort study of consecutive patients $\geq 18$ years who underwent EUSguided LAMS placement for PFCs at seven academic tertiary referral centers in the United States between January 2010 and December 2016. The study was approved by the institutional review board for human research at each participating institution, with the University of Florida serving as the central coordinating center. All patients signed procedure informed consent.
Patients were identified by searching in prospectively maintained endoscopy report-generating electronic databases and subjects' medical records were retrospectively reviewed. Data obtained from all participating centers were compiled into a central database.

PFCs were characterized by cross-sectional imaging (computed tomography and/or magnetic resonance imaging) and endoscopic ultrasound (EUS). All patients underwent EUS-guided PFC transmural drainage with LAMS (Axios, Boston Scientific, Marlborough, Massachusetts, United States) placement as previously described [4-7]. Additional interventions, including direct endoscopic necrosectomy (DEN), cavity irrigation, and/ or placement of double pigtail plastic stents (DPs) across the LAMS were performed at the discretion of the endoscopist. All endoscopic procedures were performed according to American Society of Gastrointestinal Endoscopy (ASGE) practice guideline recommendations on antibiotic prophylaxis and management of antithrombotic agents and coagulopathy $[14,15]$.

Data collection was divided into three categories: baseline, procedural, and post-procedural data. Baseline data included patient demographics, use of anticoagulation/antiplatelet therapy, and endoscopists' experience. Procedure-related data included diameter of PFC, type of LAMS (non-electrocautery vs. electrocautery-enhanced LAMS), site of cysto-enterostomy, additional interventions (e.g. DEN, DPs across LAMS), technical success and early AEs (<48 hours). Post-procedural data included findings (e.g. PFC resolution, delayed AEs) at the time of interval imaging and follow-up endoscopy.

\section{Definitions}

PFCs were defined as WON or pseudocyst (PP) based on the revised Atlanta classification [16]. Procedural success was defined as successful insertion of the LAMS during the PFC drainage. Early (defined as occurring within 48 hours after LAMS insertion) and delayed (detected during clinical follow-up with interval imaging and endoscopy) AEs were assessed based on previously established criteria by the ASGE [17]. Stent occlusion was defined as a stent with a lumen obstructed by debris as seen on imaging and/or endoscopy. Stent migration was defined as interval change in the position (inwards towards the gastrointestinal lumen or outwards towards the PFC) of the LAMS. The LAMS was inspected on follow-up endoscopy to assess for partially buried (flanges still visible) or completely buried (LAMS not visible) flanges by mucosal tissue overgrowth. Treatment success was defined as resolution of the PFC to $<3 \mathrm{~cm}$ and resolution of symptoms at the time of interval imaging following EUS-guided LAMS placement.

\section{Outcome measures}

The primary outcome of this study was to evaluate for delayed AEs after LAMS insertion for PFC (WON vs. PP) drainage. Secondary outcomes included the identification of potential risk factors for treatment failure and delayed AEs. 
- Table 1 Baseline characteristics.

\begin{tabular}{|l|c|l|l|}
\hline & Total $(\mathbf{n}=\mathbf{1 2 2})$ & WON (n=64) & Pseudocyst (n=58) \\
\hline Age, mean (SD), years & $50.9(15.4)$ & $51.8(14.4)$ & $49.6(16.4)$ \\
\hline Gender, $\mathrm{n}(\%)$ & & & \\
\hline - Female & $43(35.2)$ & $21(32.8)$ & $22(38)$ \\
\hline " Male & $79(64.8)$ & $43(67.2)$ & $36(62)$ \\
\hline Body mass index, mean (SD) $\left(\mathrm{kg} / \mathrm{m}^{2}\right)$ & $27.1(5.9)$ & $28.9(6.3)$ & $24.8(4.6)$ \\
\hline ASA grade, median (interquartile range) & $3(2-3)$ & $3(2-3)$ & $3(2-3)$ \\
\hline Antiplatelet/anticoagulation therapy, $\mathrm{n}(\%)$ & $13(10.7)$ & $8(13)$ & $5(8.8)$ \\
\hline
\end{tabular}

\section{Statistical analysis}

Descriptive statistics were calculated for all demographic, imaging, and clinical variables and were reported as mean \pm standard deviation (SD), median with ranges for continuous variables, or as a proportion for categorical variables. Univariate analysis was performed by using the Chi-Square test or the Fisher exact test for equality of proportions for categorical variables and the Student's $t$-test for continuous variables when indicated and univariate logistic regression models. Later, multivariable logistic regression analyses were performed to control for other potential confounders and risk factors in the models. Statistical analysis was implemented with the SPSS software v22 (IBM, SPSS Statistics, Armonk, New York, United States) and Stata Statistical Software version 15 (StataCorp. 2017, College Station, Texas, United States). A P value less than 0.05 was considered statistically significant.

\section{Results}

\section{Patient demographics}

A total of 122 patients (mean age 50.9 years, $68 \%$ male) with PFC (64 WON and 58 PP) underwent EUS-guided transmural drainage with a LAMS ( $\triangleright$ Table 1 ). There were no statistically significant differences in age, gender distribution, body mass index, American Society of Anesthestologists grade, or use of antithrombotic therapy between patients with WON and PP groups. The majority of cases (72/122; $60 \%)$ were performed by endoscopists who reported an experience of at least 10 cases of EUS-guided PFC drainage using a LAMS.

\section{PFC and procedural characteristics}

Procedural characteristics are summarized in $>$ Table 2. Successful insertion of a LAMS for PFC drainage (technical success) was achieved in 120 out of 122 (98.4\%) of patients; there was no difference between patients with WON (98.4\%) vs. PP $(98.3 \%)(P=1.00)$ ( $\triangleright$ Fig. 1). Mean size of the PFC was similar in patients with WON $(130.8 \pm 44 \mathrm{~mm})$ vs. $P P(95.2 \pm 48 \mathrm{~mm})(P=$ 0.58 ). The proximal stomach (fundus, cardia or body) was more commonly chosen as the location for LAMS insertion in patients with WON $(92.2 \%)$ vs. PP $(67.2 \%)(P<0.001)$. Electrocauteryenhanced LAMS $(15 \mathrm{~mm}$ wide $\times 10 \mathrm{~mm}$ long) was most commonly used in most cases.In the WON group, 23 (35.9\%) had
DEN and 7 (10.9\%) DPs placed across the LAMS during the initial endoscopic session.

\section{Early adverse events}

LAMS mis-deployment occurred in two cases in both groups. Both patients underwent successful transmural drainage with placement of DPs during the same session as rescue. Overall, early AEs occurred in 5 of 122 patients (4.1\%), with no significant difference in rates between patients with WON and PPs ( $\downarrow$ Table 2). Self-limited bleeding after stent placement was reported in one patient with WON. For the one patient with PP in whom the LAMS was mis-deployed, bleeding from the initial LAMS insertion tract required embolization of the left gastric artery to achieve hemostasis. Three cases of infection were reported, which were all treated with antibiotic therapy and ongoing drainage. There were no cases of perforation or procedure-related mortality in any of the groups.

\section{Main outcomes on interval imaging and initial follow-up endoscopy}

Nearly all patients in both the WON (61; 95\%) and PP (57; $98.3 \%$ ) groups had interval imaging and follow-up endoscopy. There was no additional information available in the electronic records to assess for clinical outcomes in the patients lost to follow-up (3 WON and 1 PP).

Findings on interval imaging (median of 4 weeks after LAMS insertion; interquartile range 3-6 weeks) are summarized in - Table 3. PFC resolution was significantly lower for patients who underwent LAMS placement for WON (62.3\%) compared to PPs $(96.5 \%)(P<0.001)$. A total of $2(3.3 \%)$ and $4(7 \%)$ cases of stent occlusion were reported for the WON and PP groups, respectively $(P=0.42)$.

Follow-up endoscopy after LAMS insertion for PFC drainage was performed at a median of 5 weeks (interquartile range 47 weeks). Stent occlusion was identified in 18 (29.5\%) and 10 (17.5\%) patients in the WON and PP groups, respectively $(P=$ $0.13)$. The overall rate of stent migration into the gastrointestinal lumen was 5.9\%; there was no differences between the WON vs. PP groups (4.9\% vs. $7.0 \% ; P=0.71)$. In one patient 
- Table 2 Procedural characteristics and immediate adverse events.

\begin{tabular}{|c|c|c|c|}
\hline & WON $(n=64)$ & Pseudocyst $(n=58)$ & $P$ value \\
\hline PFC long-axis measurement, mean (SD), (mm) & $130.8(44)$ & $95.2(48)$ & .58 \\
\hline \multicolumn{4}{|l|}{ Site of cyst enterostomy; $\mathrm{n}(\%)$} \\
\hline - Proximal stomach (fundus, cardia, body) & $59(92.2)$ & $39(67.2)$ & $<.001$ \\
\hline - Distal stomach or duodenum (antrum, bulb) & $2(3.1)$ & $17(29.3)$ & \\
\hline \multicolumn{4}{|l|}{ Diameter of LAMS; n (\%) } \\
\hline - $15 \mathrm{~mm}$ & $54(84.4)$ & $49(84.5)$ & 1.00 \\
\hline - $10 \mathrm{~mm}$ & $10(15.6)$ & $9(15.5)$ & \\
\hline \multicolumn{4}{|l|}{ Electrocautery-enhanced LAMS delivery system; n (\%) } \\
\hline - Yes & $34(53.1)$ & $41(70.7)$ & .06 \\
\hline - No & $30(46.9)$ & $17(29.3)$ & \\
\hline Procedural technical success; n (\%) & $63(98.4)$ & $57(98.3)$ & 1.00 \\
\hline \multicolumn{4}{|l|}{ Immediate adverse events; $\mathrm{n}(\%)$} \\
\hline - Bleeding & $1(1.6)$ & $1(1.7)$ & 1.00 \\
\hline - Perforation & 0 & 0 & \\
\hline - Infection & $2(3.1)$ & $1(1.7)$ & \\
\hline
\end{tabular}
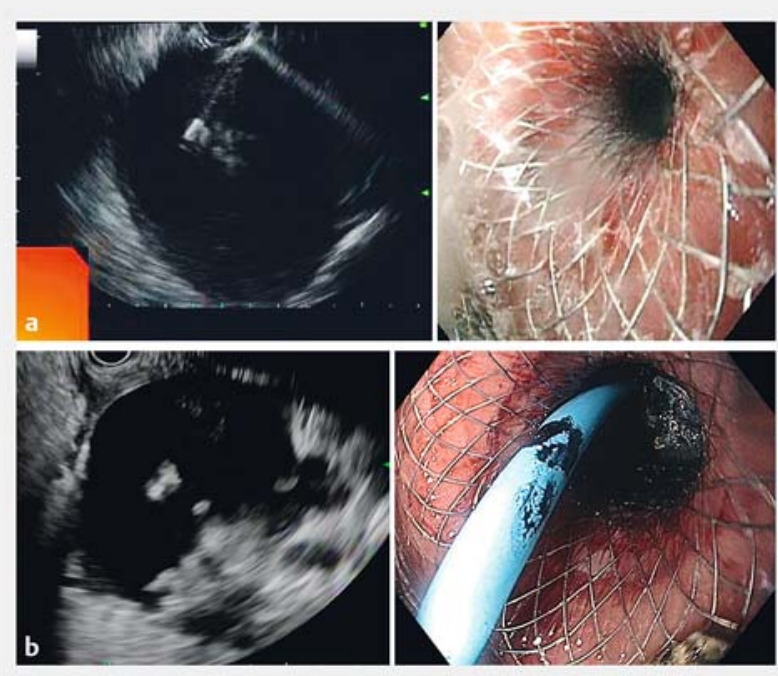

- Fig. 1 Endosonographic view of a pseudocyst. a Endoscopic view of transgastric drainage of pseudocyst via placement of a LAMS. b Endosonographic view of walled-off necrosis. Placement of DP through LAMS for WON drainage.

with a PP, the LAMS had partially migrated into the PFC but endoscopic retrieval was still accomplished by grabbing the proximal flanges. Overall, there were only two cases (one in each group) in which the proximal flanges of the LAMS were partially embedded by the mucosa from tissue overgrowth. Stent retrieval in both of these cases was successful. There were no cases of buried (LAMS flanges completely embedded by the mucosa) stent reported on follow-up endoscopy.

\section{Subgroup analysis of main outcomes in patients with WON}

Subgroup analysis of patients with WON is shown in $>$ Table 4. There was no statistically significant difference in WON resolution rates between patients who underwent DEN (69.6\%), DP through LAMS (57.1\%), or LAMS placement only (58.1\%). On follow-up endoscopy, there was no statistically significant difference in the rate of stent occlusion or migration among the three subgroups.

\section{LAMS removal and follow-up}

The LAMS was removed at the time of follow-up endoscopy in 41/64 and 47/58 patients in the WON and PP groups, respectively. Self-limited bleeding occurred in one patient with PP. One patient with WON had an esophageal tear during LAMS retrieval which was treated by placement of endoscopic clips. Both patients did not require any additional interventions and recovered uneventfully.

Thirty patients (20 WON and 10 PP) did not have the LAMS removed on initial follow-up endoscopy (\$ Fig.2). Among these patients with WON, two patients underwent surgery: one patient had an AE with infection and thereby underwent surgical cystogastrostomy and LAMS removal whereas the second patient had surgery for persistent WON and concomitant biliary stricture from chronic pancreatitis. LAMS was exchanged for DPs in three patients, whereas DEN (median 1 session; range $1-5$ sessions) was performed in the remainder 14 patients. 
- Table 3 Clinical outcomes at the time of interval imaging and follow-up endoscopy.

\begin{tabular}{|c|c|c|c|}
\hline & WON $(n=61)^{1}$ & $\mathrm{PP}(n=57)^{1}$ & $P$ value \\
\hline $\begin{array}{l}\text { Time interval from cyst enterostomy to imaging (CT/MRI); median, } \\
\text { (interquartile range) weeks }\end{array}$ & $4(2-6)$ & $4(3-6)$ & \\
\hline \multicolumn{4}{|l|}{ Findings on interval imaging; $\mathrm{n}(\%)$} \\
\hline - Resolution of PFC & $38(62.3)$ & $55(96.5)$ & $<0.001$ \\
\hline - Stent migration & 0 & 0 & \\
\hline - Stent occlusion & $2(3.3)$ & $4(7.0)$ & .42 \\
\hline - Infection & $2(3.3)$ & $2(3.5)$ & 1.00 \\
\hline $\begin{array}{l}\text { Time interval from cyst enterostomy to follow-up endoscopy; median, } \\
\text { (interquartile range) weeks }\end{array}$ & $5(2-7)$ & $6(4-8)$ & \\
\hline \multicolumn{4}{|l|}{ Findings on follow-up endoscopy; n (\%) } \\
\hline - Stent occlusion & $18(29.5)$ & $10(17.5)$ & .13 \\
\hline - Stent migration into PFC & 0 & $1(1.7)$ & .48 \\
\hline - Stent migration into the gastrointestinal tract & $3(4.9)$ & $4(7.0)$ & .71 \\
\hline - LAMS flanges partially embedded by mucosa & $1(1.6)$ & $1(1.8)$ & 1.00 \\
\hline - LAMS flanges completely embedded by mucosa (buried) & 0 & 0 & \\
\hline \multicolumn{4}{|l|}{ Adverse events associated with LAMS removal ${ }^{2}$} \\
\hline - Bleeding & 0 & $1(1.7)$ & .48 \\
\hline - Perforation & 0 & 0 & \\
\hline - Infection & 0 & 0 & \\
\hline - Pancreatitis & 0 & 0 & \\
\hline - Esophageal mucosal tear & $1(1.6)$ & 0 & 1.00 \\
\hline
\end{tabular}

- Table4 Subgroup analysis of main outcomes in patients with WON.

\begin{tabular}{|c|c|c|c|c|}
\hline & LAMS only $(n=31)$ & LAMS with DP stent(s) $(n=7)$ & LAMS with DEN $(n=23)$ & $P$ value \\
\hline \multicolumn{5}{|l|}{ Findings on interval imaging; $\mathrm{n}(\%)$} \\
\hline - Resolution of PFC & $18(58.1)$ & $4(57.1)$ & $16(69.6)$ & 0.66 \\
\hline - Stent migration & 0 & 0 & 0 & - \\
\hline - Stent occlusion & 0 & 0 & $2(8.7)$ & 0.18 \\
\hline " Infection & 0 & 0 & $2(8.7)$ & 0.18 \\
\hline \multicolumn{5}{|l|}{ Findings on follow-Up endoscopy; $\mathrm{n}(\%)$} \\
\hline - Stent occlusion & $7(22.6)$ & $5(71.4)$ & $6(26.1)$ & 0.03 \\
\hline - Stent migration into PFC & 0 & 0 & 0 & - \\
\hline - Stent migration into the gastrointestinal tract & $1(3.2)$ & 0 & $2(8.7)$ & 0.53 \\
\hline - LAMS flanges partially embedded by mucosa & 0 & 0 & $1(4.3)$ & 0.43 \\
\hline $\begin{array}{l}\text { - LAMS flanges completely embedded by } \\
\text { mucosa (buried) }\end{array}$ & 0 & 0 & 0 & - \\
\hline
\end{tabular}




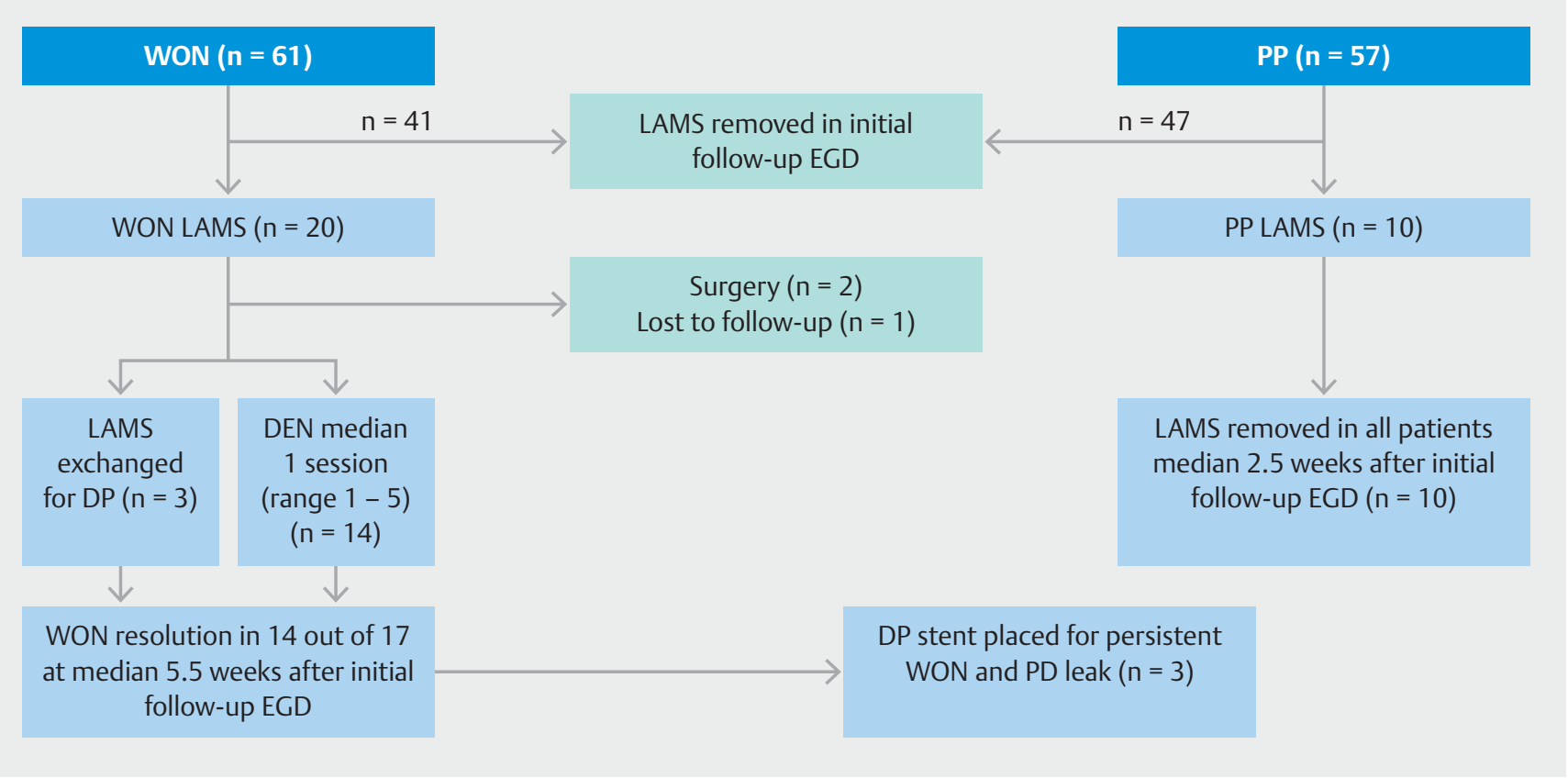

- Fig. 2 Flow chart of patient in whom LAMS was not initially removed at the time of initial follow-up endoscopy.

Overall, WON resolution was noted in 14 out of 17 patients at a median of 5.5 weeks (range $4-12$ weeks) since their initial follow-up endoscopy. For those patients in whom WON did not resolve, three had DPs placed due to pancreatic duct leak.

For patients with PP, subsequent stent removal $(n=10)$ was performed at a median of 2.5 weeks (range 1 to 11 weeks) after their initial follow-up endoscopy. Stent removal was uneventful in all patients, even though one of LAMS was found partially embedded. In one patient, the stent had spontaneously migrated into the stomach.

\section{Predictors of treatment failure and stent occlusion}

Univariable and multivariable logistic regression was performed to identify predictors of treatment failure (persistent PFC on interval imaging) and stent occlusion (the most commonly identified delayed adverse event in both the WON and PP cohorts). Factors, including patient demographics (age, gender, ASA score), endoscopist experience (>10 cases; yes vs. no), procedural characteristics (use of electrocautery-enhanced LAMS, Iocation and diameter of LAMS, placement of additional stents, DEN, size of PFC), were included. Use of electrocautery-enhanced LAMS was the only factor strongly associated with treatment failure in the EUS-guided transmural drainage of WONs on both univariable $(\mathrm{OR}=13.15,95 \% \mathrm{Cl}$ : $3.33-51.82$, $P=0.02)$ and multivariable $(\mathrm{OR}=35.7,95 \% \mathrm{Cl}: 4.9-255.56, P=$ 0.01 ) analysis ( $\triangleright$ Table 4 ). None of the above mentioned factors included in the univariable and multivariable analysis significantly correlated with treatment failure of PPs ( $\downarrow$ Table 5 ). There were no factors predictive of risk of stent occlusion in WON or PPs ( $>$ Table 6 ).

\section{Discussion}

Endoscopic transmural drainage is now standard practice for initial management of symptomatic PFCs, along with adjunct surgical and percutaneous approaches. In recent years, development of the LAMS device has further consolidated this practice by overcoming some of the inherent technical limitations associated with use of conventional tubular fully covered metal stents and/or DPs [18]. However, in spite of the accumulating data on the efficacy and safety of LAMS placement for PFC drainage, there has been an increasing concern regarding risk for delayed AE associated with the indwelling LAMS. In this multicenter study, we demonstrate that EUS-guided LAMS placement for PFC is effective and safe, delayed $A E$ are rare, and the use of electrocautery-enhanced LAMS was the sole predictor of failure of resolution of WON.

Several recent studies have reported on the occurrence of delayed AE associated with LAMS for PFC drainage. In a trial of 21 patients randomized to LAMS vs. DPs for PFC drainage, Bang et al reported a higher than anticipated AE rate with LAMS [13]. Stent-related $A E$, including delayed bleeding $(n=3)$, buried stent $(n=2)$, and stent-induced biliary stricture $(n=1)$, were observed in $50 \%$ of the patients randomized to LAMS $(n=12)$ compared with none in the DP stent cohort; with all AEs being observed 3 weeks after index intervention. The authors postulated that risk of delayed bleeding is presumably secondary to mechanical abrasion/impingement of adjacent vasculature by the flanges of the LAMS upon collapse/resolution of the PFC. In addition to delayed bleeding, there have also been a few case reports describing the phenomenon of buried stent syndrome, in which the flanges of the LAMS are deeply embedded within the mucosa due to tissue overgrowth, thereby complicating stent removal [8-11]. Based on these findings, Bang et 
- Table 5 Uni- and multivariable analysis evaluating predictors of treatment failure of WON and PPs.

\begin{tabular}{|c|c|c|c|c|c|c|c|c|}
\hline \multirow{3}{*}{ Clinical variable } & \multicolumn{4}{|l|}{ WON } & \multicolumn{4}{|l|}{ PPs } \\
\hline & \multicolumn{2}{|c|}{ Univariable analysis } & \multicolumn{2}{|c|}{ Multivariable analysis } & \multicolumn{2}{|c|}{ Univariable analysis } & \multicolumn{2}{|c|}{ Multivariable analysis } \\
\hline & OR $(95 \% \mathrm{Cl})$ & $P$ value & OR $(95 \% \mathrm{Cl})$ & $P$ value & OR $(95 \% \mathrm{Cl})$ & $P$ value & OR $(95 \% \mathrm{Cl})$ & $P$ value \\
\hline Age (years) & $\begin{array}{l}1.03 \\
(1.00-1.07)\end{array}$ & 0.12 & $\begin{array}{l}1.03 \\
(0.99-1.09)\end{array}$ & 0.17 & $\begin{array}{l}1.04 \\
(0.99-1.1)\end{array}$ & 0.17 & $\begin{array}{l}1.06 \\
(0.98-1.14)\end{array}$ & 0.15 \\
\hline Gender (male vs. female) & $\begin{array}{l}0.80 \\
(0.27-2.42)\end{array}$ & 0.70 & $\begin{array}{l}0.34 \\
(0.06-1.80)\end{array}$ & 0.20 & $\begin{array}{l}1.15 \\
(0.19-6.9)\end{array}$ & 0.87 & $\begin{array}{l}0.48 \\
(0.04-6.43)\end{array}$ & 0.58 \\
\hline ASA score (3/4 vs. $1 / 2$ ) & $\begin{array}{l}0.98 \\
(0.34-2.81)\end{array}$ & 0.97 & $\begin{array}{l}0.86 \\
(0.17-4.24)\end{array}$ & 0.73 & $\begin{array}{l}2.7 \\
(0.29-25)\end{array}$ & 0.39 & - & - \\
\hline $\begin{array}{l}\text { Endoscopist experience } \\
\text { ( } \geq 10 \text { cases) }\end{array}$ & $\begin{array}{l}1.1 \\
(0.41-3.03)\end{array}$ & 0.84 & $\begin{array}{l}2.72 \\
(0.44-17.01)\end{array}$ & 0.23 & $\begin{array}{l}0.37 \\
(0.07-2.1)\end{array}$ & 0.25 & $\begin{array}{l}0.26 \\
(0.02-2.9)\end{array}$ & 0.27 \\
\hline DEN (yes vs. no) & $\begin{array}{l}0.51 \\
(0.17-1.49)\end{array}$ & 0.22 & $\begin{array}{l}0.34 \\
(0.06-2.04)\end{array}$ & 0.24 & & & & \\
\hline Additional stents (yes vs. no) & $\begin{array}{l}0.80 \\
(0.27-2.4)\end{array}$ & 0.69 & $\begin{array}{l}1.83 \\
(0.30-11.3)\end{array}$ & 0.52 & & & & \\
\hline $\begin{array}{l}\text { Electrocautery-enhanced } \\
\text { LAMS }\end{array}$ & $\begin{array}{l}13.15 \\
(3.33-51.82)\end{array}$ & 0.02 & $\begin{array}{l}35.7 \\
(4.90-255.56)\end{array}$ & 0.01 & $2(0.22-20)$ & 0.53 & - & - \\
\hline PFC size (mm) & $\begin{array}{l}1.01 \\
(1.0-1.02)\end{array}$ & 0.25 & $1.02(1.0-1.04)$ & 0.15 & $\begin{array}{l}1.01 \\
(1-1.03)\end{array}$ & 0.26 & $\begin{array}{l}1.01 \\
(0.99-1.03)\end{array}$ & 0.19 \\
\hline $\begin{array}{l}\text { Location of LAMS (proximal } \\
\text { vs. distal) }\end{array}$ & $\begin{array}{l}0.68 \\
(0.04-11.31)\end{array}$ & 0.79 & $\begin{array}{l}0.32 \\
(0.001-309.92)\end{array}$ & 0.75 & & & & \\
\hline $\begin{array}{l}\text { Diameter of LAMS ( } 15 \text { mm; } \\
\text { yes vs. no) }\end{array}$ & $\begin{array}{l}4.69 \\
(0.53-41.50)\end{array}$ & 0.17 & $\begin{array}{l}5.24 \\
(0.20-135.65)\end{array}$ & 0.32 & & & & \\
\hline
\end{tabular}

WON, walled-off necrosis; PP, pseudocyst; ASA, American Society of Anesthesiologists; DEN, direct endoscopic necrosectomy; LAMS, lumen-apposing metal stent; PFC, peripancreatic fluid collection

- Table 6 Univariable and multivariable analysis evaluating predictors of stent occlusion in WON and PPs.

\begin{tabular}{|c|c|c|c|c|c|c|c|c|}
\hline \multirow{3}{*}{ Clinical variable } & \multicolumn{4}{|l|}{ WON } & \multicolumn{4}{|l|}{ PPs } \\
\hline & \multicolumn{2}{|c|}{ Univariable analysis } & \multicolumn{2}{|c|}{ Multivariable analysis } & \multicolumn{2}{|c|}{ Univariable analysis } & \multicolumn{2}{|c|}{ Multivariable analysis } \\
\hline & OR $(95 \% \mathrm{Cl})$ & $P$ value & OR $(95 \% \mathrm{Cl})$ & $P$ value & OR $(95 \% \mathrm{Cl})$ & $P$ value & OR $(95 \% \mathrm{Cl})$ & $P$ value \\
\hline Age (years) & $\begin{array}{l}1 \\
(0.96-1.03)\end{array}$ & 0.39 & $\begin{array}{l}0.97 \\
(0.92-1.02)\end{array}$ & 0.37 & $\begin{array}{l}1.04 \\
(0.99-1.1)\end{array}$ & 0.17 & $\begin{array}{l}1.02 \\
(0.9-1.1)\end{array}$ & 0.86 \\
\hline Gender (male vs. female) & $\begin{array}{l}0.97 \\
(0.3-3.1)\end{array}$ & 0.95 & $0.63(0.15-2.7)$ & 0.54 & $\begin{array}{l}1.25 \\
(0.19-6.9)\end{array}$ & 0.87 & $\begin{array}{l}0.21 \\
(0.02-3.1)\end{array}$ & 0.26 \\
\hline ASA score (3/4 vs. $1 / 2)$ & $\begin{array}{l}2.1 \\
(0.65-6.8)\end{array}$ & 0.21 & $4.1(0.82-20.2)$ & 0.09 & $\begin{array}{l}2.7 \\
(0.29-25)\end{array}$ & 0.39 & - & - \\
\hline $\begin{array}{l}\text { Endoscopist experience } \\
\text { ( } \geq 10 \text { cases) }\end{array}$ & $\begin{array}{l}4.2 \\
(1.3-13.1)\end{array}$ & 0.01 & $14.5(2.1-100)$ & 0.07 & $\begin{array}{l}0.37 \\
(0.07-2.1)\end{array}$ & 0.25 & $\begin{array}{l}0.34 \\
(0.02-5.3)\end{array}$ & 0.44 \\
\hline DEN (yes vs. no) & $\begin{array}{l}0.61 \\
(0.19-1.9)\end{array}$ & 0.39 & $1(0.1-9.3)$ & 0.99 & & & & \\
\hline Additional stents (yes vs. no) & $\begin{array}{l}1.8 \\
(0.58-5.5)\end{array}$ & 0.31 & $5(0.8-29.3)$ & 0.07 & & & & \\
\hline $\begin{array}{l}\text { Electrocautery-enhanced } \\
\text { LAMS }\end{array}$ & $\begin{array}{l}1.28 \\
(0.4-3.7)\end{array}$ & 0.64 & $3.6(0.9-14.2)$ & 0.06 & $\begin{array}{l}2 \\
(0.22-20)\end{array}$ & 0.53 & - & - \\
\hline PFC size $(\mathrm{mm})$ & $\begin{array}{l}1.01 \\
(0.98-1.01)\end{array}$ & 0.52 & $0.9(0.98-1.01)$ & 0.98 & $\begin{array}{l}1.01 \\
(1-1.03)\end{array}$ & 0.26 & $\begin{array}{l}1.02 \\
(0.99-1.05)\end{array}$ & 0.28 \\
\hline
\end{tabular}

WON, walled-off necrosis; PP, pseudocyst; ASA, American Society of Anesthesiologists; DEN, direct endoscopic necrosectomy; LAMS, lumen-apposing metal stent; PFC, peripancreatic fluid collection 
al. recommended that a CT scan should be obtained at the threshold of 3 weeks and LAMS removed if the PFC has resolved.

In our multicenter study, 118 patients with LAMS underwent interval imaging and repeat endoscopy at a median of 4 and 5 weeks, respectively. Overall, there was only one case of delayed self-limited bleeding at the time of stent removal on follow-up endoscopy. In two patients, the LAMS was partially buried but there were no difficulties associated with stent retrieval. Our findings demonstrate that incidence of delayed bleeding or buried stent at the time of follow-up is very low. As such, performing a CT scan at 3 weeks in all patients treated with LAMS may not necessarily reduce the potential risk of these delayed AEs, but increase costs associated with early imaging, as many of these PFCs, particular WON, may still be persistent. Indeed, in our study, only $62.3 \%$ of patients with WON had radiographic resolution on CT or MRI at a median of 4 weeks, as compared to resolution in nearly all cases of PP (96.5\%). The lower resolution rate of WON as compared to PP would appear to be intuitively associated with the degree of solid debris within the WON. Indeed, a recent study in abstract form demonstrated that assessment of the degree of solid material within a WON on cross-sectional imaging accurately predicted clinical outcomes of endoscopic drainage of WON with LAMS [19]. Future prospective studies stratifying WON based on degree of solid material within the collection and controlling for the type of intervention (e.g. LAMS with DEN, LAMS with DP, LAMS alone) are urgently needed to better define the best approach for these patients. Overall, while early imaging ( 3 weeks) may be effective for PPs as most of these have resolved within this period, the optimum time for repeat imaging and stent removal in patients with WON remains to be determined.

Multivariable analysis in the current study demonstrated that use of an electrocautery-enhanced delivery system was the only predictor of failure of resolution of WON, but not PPs. We can only speculate about possible explanations for these findings. Traditionally, when performing endoscopic PFC drainage, the fistulous tract is dilated to aid advancement of the delivery system and stent placement. With the electrocautery-enhanced LAMS, the tip on the delivery system permits passage of the deployment device, obviating the need for prior fistulous tract dilation. It is conceivable that initial tract dilation prior to insertion of the non-electrocautery enhanced LAMS may facilitate accommodation and quicker stent expansion, thereby prompting drainage of necrotic debris when compared to the one-step approach with electrocautery-enhanced LAMS.Certainly, further prospective controlled studies comparing outcomes between these two types of LAMS delivery systems for WON drainage are needed to further clarify the significance of these initial results.

In our study, stent occlusion was the most common delayed $\operatorname{AE}(n=28 ; 23.7 \%)$ on follow-up endoscopy. Even though by definition PPs are devoid of solid debris, stent occlusion was noted in $17.5 \%$ of our patients. Our findings are consistent with those recently reported by Aburajab et al, in which $17 \%$ patients with PP treated with LAMS were complicated by infection presumably from stent occlusion from food debris [20].On our multi- variable analysis, neither DEN nor DPs through the LAMS were predictors of stent occlusion. In spite of its larger diameter when compared to DPs, and its anchoring flanges which facilitate DEN and reduce risk of stent migration when compared to conventional FCSEMS, the ideal endoscopic approach with LAMS for WON is not clear. Whether a "step-up" approach, placement of DPs or nasocystic drain through LAMS, or scheduled DEN, may promote WON resolution and reduce incidence of stent occlusion remain to be determined [21-23].

This study has several strengths. In this multicenter experience, we demonstrate that risk of delayed bleeding or buried LAMS is very low at the time of follow-up. These observations have direct clinical implications for our approach to PFC management following LAMS insertion. Given that nearly all PPs resolve during this time interval, repeat imaging at 4 weeks (or perhaps even earlier) followed by LAMS removal is both safe and cost-effective. Conversely, we demonstrate that many WON persist during this time frame. As such, CT or MRI could be reserved for those patients in which complications are suspected, whereas transabdominal ultrasound, a more cost-effective approach, may be used to triage timing of repeat endoscopic interventions (DEN, stent removal, etc). Given the current wide variation in practice, particularly in treatment of WON, randomized studies are urgently needed to help establish best practice consensus [24].

We also acknowledge the limitations of this study. This multicenter study was conducted in tertiary centers and thus our results may not be generalizable. The study design was retrospective and thereby limited by its uncontrolled design and potential for selection bias. Furthermore, timing and type of interventions (e.g. balloon dilation at the time of LAMS placement, DEN and/or DP placement, diameter of the LAMS used) were not controlled as these were performed at the discretion of the endoscopists.Nonetheless, these factors were included in our logistic regression and were not found to be significantly associated with our main outcomes. We also recognize that while this represents a relatively large study of this nature (LAMS for PFC), the absolute small number of patients could have still precluded detection of potential importance differences in outcomes, particularly in subgroup analysis of patients with additional interventions for WON. Furthermore, selection bias (e.g. more severe cases may have undergone more aggressive measures of endoscopic drainage) may have also accounted for the apparent lack of WON resolution rates among the subgroups (e.g. LAMS with DEN, DP through LAMS, and LAMS only) and thereby these results should be interpreted with caution. We also recognize that our rate of WON resolution (62.3\%) at the time of interval imaging is lower than what has been previously reported on LAMS for WON $[6,7]$. These apparent differences may be in part associated with the heterogeneity of patient populations (e.g. extent of necrotic debris, size of WON) and type of interventions (e.g. timing and number of DEN, DPs placed) among the various studies. If we include the patients with WON in our study who had resolution after additional interventions (DEN through LAMS) following interval imaging $(n=11)$, the treatment success was actually $80.3 \%$ and thereby consistent with those recorded in the literature. 


\section{Conclusion}

In summary, EUS-guided LAMS placement for drainage of both WON and PPs is associated with a low incidence of delayed bleeding or buried stent syndrome. While nearly all PPs resolve at 4 weeks permitting LAMS removal shortly thereafter, many WON persist, with use of electrocautery-enhanced LAMS being the sole predictor of failure of resolution. Future controlled studies are needed to further define the optimal interval for interval imaging and endoscopic therapy for patients with WON.

WON, walled-off necrosis; CT, computed tomography; MRI, magnetic resonance imaging; PP, pseudocyst; PFC, peripancreatic fluid collection; LAMS, lumen-apposing metal stent

\section{Competing interests}

Dr Wang has received research funding from Cook Medical. Drs Draganov and Aslanian are consultants for Boston Scientific. Dr Buscaglia has been a speaker for Boston Scientific and Abbvie within the past 12 months.

\section{References}

[1] Varadarajulu S, Bang JY, Sutton BS et al. Equal efficacy of endoscopic and surgical cystogastrostomy for pancreatic pseudocyst drainage in a randomized trial. Gastroenterology 2013; 145: 583-590

[2] Van Brunschot S, van Grinsven J, van Santvoort HC et al. Endoscopic or surgical step-up approach for infected necrotizing pancreatitis: a multicentre randomized trial. Lancet 2018; 39: 51-58

[3] Yang D, Draganov PV. EUS-Guided transmural drainage of pancreatic fluid collections: keeping it close. Pancreat Disord Ther 2016; 6: e144

[4] Shah RJ, Shah JN, Waxman I et al. Safety and efficacy of endoscopic ultrasound-guided drainage of pancreatic fluid collections with lumen-apposing covered self-expanding metal stent. Clin Gastroenterol Hepatol 2015; 13: $747-752$

[5] Siddiqui AA, Adler DG, Niejo J et al. EUS-guided drainage of peripancreatic fluid collections and necrosis using a novel lumen-apposing stent: a large retrospective multicenter U.S. experience (with videos). Gastrointest Endosc 2016; 83: 699- 707

[6] Sharaiha RZ, Tyberg A, Khashab MA et al. endoscopic therapy with lumen-apposing metal stents is safe and effective for patients with pancreatic walled-off necrosis. Clin Gastroenterol Hepatol 2016; 14 : $1797-1803$

[7] Siddiqui AA, Kowalski TE, Loren DE et al. Fully covered self-expanding metal stents versus lumen-apposing fully covered self-expanding metal stent versus plastic stents for endoscopic drainage of pancreatic walled-off necrosis: clinical outcomes and success. Gastrointest Endosc 2017; 85: $758-765$

[8] Fabbri C, Luigiano C, Marsico $\mathrm{M}$ et al. A rare adverse even resulting from the use of a lumen-apposing metal stent for drainage of a pancreatic fluid collection: "the buried stent". Gastrointest Endosc 2015; 82: $585-587$
[9] Seerden TC, Vleggaar FP. Endoscopic removal of buried lumen-apposing metal stents used for cystogastrostomy and cholecystogastrostomy. Endoscopy 2016; 48: (Suppl. 01): E179

[10] Altonbary AY, Hakim H. The buried stent: a rare complication of endoscopic ultrasound-guided pancreatic necrosectomy using a lumen-apposing metal stent. Endoscopy 2017; 49: E84-E85

[11] Sanchez-Ocana R, Peňas-Herrero I, Santons-Santamarta F et al. EUSguided removal of a buried lumen-apposing metal stent caused by delayed inward migration after cyst-gastrostomy. Gastrointest Endosc 2017; 86: 229

[12] Brimhall BB, Han S, Tatman PD et al. Lumen apposing metal stents compared with double pigtail plastic stents in the management of pancreatic pseudocysts and walled-off necrosis: a 5 year single center study. Gastrointest Endosc 2017; 85: AB90

[13] Bang JY, Hasan M, Navaneethan U et al. Lumen-apposing metal stents (LAMS) for pancreatic fluid collection (PFC) drainage: may not be business as usual. Gut 2016; 66: 2054-2056

[14] ASGE Standards ofPractice Committee, Khashab MA, Chithadi KV et al. Antibiotic prophylaxis for Gl endoscopy. Gastrointest Endosc 2015; 81: $81-89$

[15] Anderson MA, Ben-Menachem T, Gan SI et al. ASGE Standards of Practice Committee. management of antithrombotic agents for endoscopic procedures. Gastrointest Endosc 2009; 70: 1060 - 1070

[16] Banks PA, Bollen TL, Dervenis C et al. Classification of acute pancreatitis - 2012: revision of the Atlanta classification and definitions by international consensus. Gut 2012; 62: 102 - 111

[17] Cotton PB, Eisen GM, Aabakken L et al. A lexicon for endoscopic adverse events: report of an ASGE workshop. Gastrointest Endosc 2010; 71: $450-454$

[18] Penn DE, Draganov PV, Wagh MS et al. prospective evaluation of the use of fully covered self-expanding metal stents of EUS-guided transmural drainage of pancreatic pseudocysts. Gastrointest Endosc 2012; 76: $679-684$

[19] Siddiqui A, Li Z, Kowalski TE et al. Computed tomography or magnetic resonance imaging characteristics of pancreatic walled-off necrosis accurately predicts the clinical outcomes of endoscopic transmural drainage using lumen-apposing fully covered self-expanding metal stents: a multicenter experience. Gastrointest Endosc 2017; 85: AB216

[20] Aburajab M, Smith Z, Khan A. Safety and efficacy of lumen-apposing metal stents with and without simultaneous double-pigtail plastic stents for draining pancreatic pseudocysts. Gastrointest Endosc 2018; 87: $1248-1255$

[21] Rana SS, Sharma V, Sharma R et al. Endoscopic ultrasound guided transmural drainage of walled off pancreatic necrosis using a "stepup" approach: a single center experience. Pancreatology 2017; 17: $203-208$

[22] Cosgrove N, Loren DE, Siddiqui AA et al. Effect of scheduled vs. "step up" necrosectomy for walled-off pancreatic necrosis on hospital admission and necrosis resolution. Gastrointest Endosc 2017; 85: AB101

[23] Lakhatakia S, Basha J, Talukdar R et al. Endoscopic "step-up approach" using a dedicated biflanged metal stent reduces the need for direct necrosectomy in walled-off necrosis (with videos). Gastrointest Endosc 2016; 85: $1243-1252$

[24] Guo J, Saftoui A, Vilmann P et al. A multi-institutional consensus on how to perform endoscopic ultrasound-guided peri-pancreatic fluid collection drainage and endoscopic necrosectomy. Endosc Ultrasound 2017; 6: $285-291$ 